\title{
Malcolm Walmsley
}

\author{
Charles Malcolm Walmsley passed away on 1 May 2017. He made numerous fundamental contributions \\ to the physics and chemistry of star formation and the interstellar medium. He was an exceptional \\ scientist, a highly esteemed colleague and a true gentleman.
}

A fter growing up in India and Ireland, Malcolm Walmsley studied physics at Trinity College in Dublin. In 1964, after receiving his master's degree, he moved to the newly founded University of California, San Diego, where he found a stimulating environment among supervisors Geoffrey Burbidge and William Mathews. His 1969 dissertation was on the theory of extragalactic radio sources and planetary nebulae. In La Jolla, Malcolm encountered Michael Grewing, a meeting that would determine the start of his career in astronomy.

At Michael's recommendation, Malcolm joined a new department at the Max Planck Institute for Radio Astronomy (MPIfR) in Bonn, Germany. Initially focusing his work on the energetics of the interstellar medium (ISM), he naturally became involved in radio astronomy, taking advantage of the MPIfR's newly finished 100-m telescope at Effelsberg. Among many things, he studied the radio recombination lines from carbon that had been found in interstellar cloud regions detached from the hottest, most luminous stars. In 1974, with Dy Hoang-Binh, Malcolm inferred the properties of their emission regions, anticipating the discovery of photodissociation regions long before this term was introduced and they were recognized as an important component of the ISM. Some years later, Malcolm extended his research on carbon recombination lines to very low frequencies. This work is now experiencing a renaissance as modern lowfrequency radio telescopes such as LOFAR (Low Frequency Array) allow new access to these absorption lines.

After a few years in Bonn, Malcolm became a full staff member, with the expectation that he would 'work on molecules'. And this he really did! For more than two decades he extensively used the Effelsberg 100-m telescope for molecular spectroscopy - combining his great theoretical expertise with the new telescope was a recipe for success. Malcolm joined forces with Ed Churchwell and Gisbert Winnewisser, targeting, among others, long carbon chain molecules (the cyanopolyynes). This led, in 1979, to

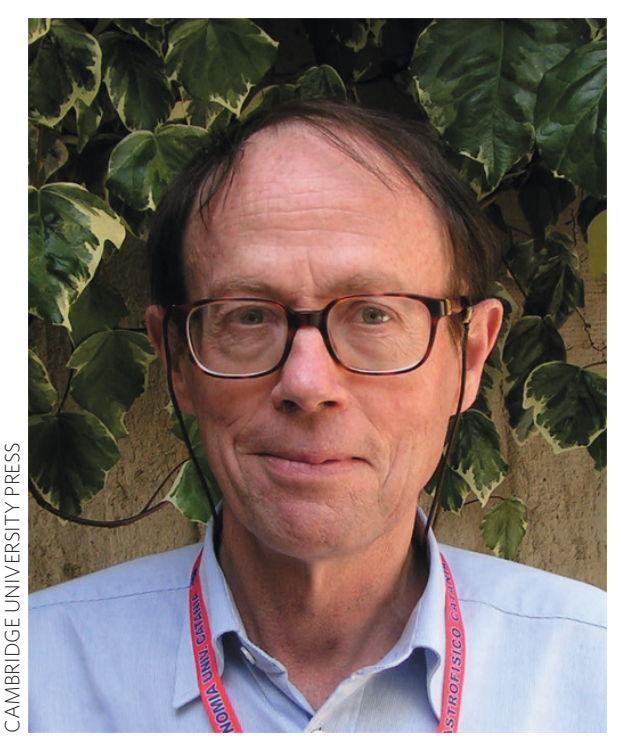

Charles Malcolm Walmsley (1941-2017)

the discovery of the Taurus Molecular Cloud 1, which has become one of the most astrochemically interesting sources. Another famous paper from this time, with his first student Hans Ungerechts, firmly established the ammonia molecule as a molecular cloud thermometer.

In 1986, sadly, Malcolm's wife Colette succumbed to cancer. Malcolm found solace in his two children and his work. Around the same time, Malcolm became interested in newly detected maser lines from methanol, which became the dissertation topic of one of the authors (K.M.). As well as observing these masers, Malcolm conducted calculations in order to understand their excitation. This led him to the prediction of anti-maser action in a methanol line at $12.2 \mathrm{GHz}$, which appears as enhanced absorption against the cosmic microwave background. He later detected this in cold dark clouds, a discovery he always cherished. In contrast, in star forming regions this line and several others show strong maser action, and methanol masers have become an unambiguous tracer of embedded high-mass protostellar objects, spawning an entire industry.
Some years later, in collaboration with one of the authors (R.C.), Malcolm developed a theoretical model that explained the excitation of interstellar $\mathrm{OH}$ masers. While not a 'maser person' per se, Malcolm's contributions to this field have been fundamental and lasting, so much so that he was invited to give the summary talk of a topical International Astronomical Union symposium on masers that is to take place in Sardinia later this year. Now, it will be dedicated to his memory.

A very productive collaboration of Malcolm's brought his student Peter Schilke into contact with two theoreticians, David Flower and Guillaume Pineau des Forêts. Over two decades they published a series of highly interesting theoretical astrochemical studies grounded by observations. These covered a wide variety of themes including a recalibration of the ammonia thermometer, the firm establishment of the $\mathrm{SiO}$ molecule as a tracer of interstellar shock waves, and deuterium fractionation.

Malcolm was a staff member at the MPIfR from 1969 to 1994 , but moved to take up a professorship at the University of Cologne. And from there, he relocated to the Arcetri Astrophysical Observatory in Florence with his second wife Antonella Natta. Here, he quickly adapted to the new environment, learning Italian, his fourth language. He pursued his research on the physics and chemistry of star forming regions, with special attention to the problem of deuterium fractionation and depletion in cold pre-stellar cores in collaboration with Paola Caselli. He also extended his interests to infrared wavelengths. However, he never relinquished his activity as a radio astronomer. At this time he made important contributions to searches for circumstellar disks around massive (proto)stars with the Plateau de Bure interferometer and, recently, the Atacama Large Millimeter/ submillimeter Array.

Malcolm returned to the MPIfR in 2001/2002 to help its Millimeter and Submillimeter Astronomy department in difficult times, when one of the 
authors (K.M.), its director, was struck by severe illness. This will always be gratefully remembered.

Even after formally retiring in 2008 , Malcolm remained an active member of the Stars and Star Formation group of the Arcetri Observatory, which he had led for several years. After Antonella's retirement in 2011, the couple progressively moved the centre of their activities to the Dublin Institute for Advanced Studies, in Ireland. Already some years earlier, Malcolm had become a scientific editor of Astronomy \& Astrophysics, one of the major journals of the field. His grasp of the science, together with his strong belief in doing the best possible job, made Malcolm a truly outstanding editor. For many, he reinforced the belief in the value of the peer review system. Malcolm adamantly, but diplomatically, enforced scientific quality. Often after a referee was happy with a manuscript's revisions, Malcolm's 'final' suggestions ("But, furthermore I wish you to consider...") prompted a significant amount of extra work, frequently more than was suggested by the referee.

What frequently amazed us was Malcolm's instant recall of any topic that he had ever worked on. Just last year he made substantial contributions to an article on methanol masers that was published 30 years after his first one on the topic! While being a supremely competent and successful scientist, Malcolm always shared his ideas freely. He was a very modest and selfless person who actually flinched when addressed as Professore in Florence. His advice helped many and was crucial for young scientists early in their careers. This is especially true for the students that he supervised.

Malcolm has shaped large areas of interstellar medium and star formation research and left a lasting legacy. Above all, Malcolm will always be in our hearts and thoughts as a truly remarkable human being.

KARL MENTEN \& RICCARDO CESARONI Karl Menten is at the Max-Planck-Institut für Radioastronomie, Auf dem Hügel 69, D-53121 Bonn, Germany. Riccardo Cesaroni is at the INAF, Osservatorio Astrofisico di Arcetri, Largo Enrico Fermi 5,

I-50125 Firenze, Italy. e-mail:kmenten@mpifr.de; cesa@arcetri.inaf.it 